\title{
Plasmodium vivax-infected Red Blood Cell
}

National Cancer Institute

\section{Source}

National Cancer Institute. Plasmodium vivax-infected Red Blood Cell. NCI Thesaurus.

Code C155655.

A preparation of red blood cells (RBCs) that have been infected with the malaria parasite Plasmodium vivax (P. vivax), with potential immunomodulating and antineoplastic activities. Upon administration of the P. vivax-infected RBCs, the P. vivax infection stimulates host immune responses against the P. vivax-infected RBCs. This also stimulates both innate and adaptive anti-tumor immune responses. This leads to the production of cytokines, including interferon-gamma (IFN-g) and tumor-necrosis factor alpha (TNF-a), the proliferation and activation of natural killer (NK) cells, dendritic cells (DCs), CD4-positive T-lymphocytes, and cytotoxic T-lymphocytes (CT Ls). This results in the inhibition of tumor cell proliferation, induction of tumor cell apoptosis, and prevents angiogenesis. 